\title{
Quo vadis Verschuldung? Szenarien zur Haushaltspolitik des Bundes
}

von Uwe Wagschal

\begin{abstract}
Sozialwissenschaftiche Analysen orientieren sich meist an der Vergangenheit, doch sollte die Disziplin auch in der Lage sein, künftige Entwicklungen abzuschätzen. Hierfür stehen unterschiedliche Methoden zur Verfügung: Prognosen, Planungsverfahren, Simulationen und Szenarioanalysen. Auf der Basis dieser Verfahren untersucht der Beitrag die Frage, ob der Bund - wie von der Verfassung vorgeschrieben - ab dem Jahr 2016 einen ausgeglichenen Haushalt vorlegen kann. Prognostiziert wird, wie sich die Finanzen des Bundes in den kommenden vier Jahren entwickeln könnten. Sowohl die Auswertungen des Domar-Modells als auch die Analyse der mittelfristigen Finanzplanung weisen auf ein Erreichen der Zielmarke hin. Komplexer fallen die vier diskutierten Szenarien aus, die unterschiedliche Ausprägungen bedeutender exogener wie endogener Einflussfaktoren berücksichtigen. Als zentrale exogene Variablen werden das Wirtschaftswachstum, die Zinsentwicklung, der Wechselkurs und die Arbeitslosigkeit angesehen. Endogene Faktoren sind hingegen der Budgetprozess, die politische Stärke des Finanzministers und die Koordinierung der Wirtschaftspolitik.
\end{abstract}

Social science analyses are predominantly ex-post oriented. However, the discipline should also seek to predict future developments. Different methods are applicable for such forecasts: prognoses, planning, simulations and scenarios. On the basis of these approaches, this contribution looks at the question whether Germany's government is going to achieve a balanced budget by 2016 (as required by the constitution) by analyzing the development of federal public finances across the upcoming electoral term. According to the Domar model as well as the official medium-term financial planning documents, this objective can be achieved. The comparatively complex scenario-based analyses, however, yield more nuanced results, taking into account important exogenous and endogenous factors. Economic growth, interest and exchange rates, and the unemployment level are the key exogenous factors, whereas the budget process, the political clout of the finance minister, and the co-ordination of economic policies constitute the endogenous variables.

\section{Einleitung: Sind in der Politischen Ökonomie nur ex-post- Analysen sinnvoll?}

Mit seiner wegweisenden Studie zur sozialdemokratischen Krisenpolitik Ende der 1970er Jahre legte Fritz Scharpf die Grundlagen für eine spieltheoretische 
Analyse makroökonomischer Koordinierung in der Wirtschaftspolitik, die das Verhalten der wichtigsten Akteure (Staat, Gewerkschaften, Arbeitgeber und Notenbank) modelliert. An anderer Stelle führte Scharpf diese Überlegungen weiter und stellte sie anhand des rational-choice-Ansatzes auf eine theoretische Basis. ${ }^{1}$ Damit begründete er den akteurzentrierten Institutionalismus. Der große Charme der Scharpfschen Analyse liegt dabei in einer stimmigen und plausiblen ex-post-Erklärung wirtschaftspolitischer Entwicklungen in Deutschland, Großbritannien, Österreich und Schweden.

Nun will bzw. sollte sozialwissenschaftliche Forschung auch künftige Entwicklungen und Tendenzen vorhersagen können (und wollen), wobei „Prognosefehler" - auch im statistischen Sinne - natürlich und zu erwarten sind. Die unterschiedliche Logik eines diachronen bzw. synchronen Vergleichs hat Steffen Ganghof herausgearbeitet, der zwischen einer X-zentrierten und einer Yzentrierten Forschungsstrategie unterscheidet. ${ }^{2}$ Der X-zentrierten Forschung ist dabei eine ,Zukunftsperspektive“ inhärent. So fragen X-zentrierte Forschungsansätze: Wozu führt X (also die unabhängige(n) Variable(n))? Im Forschungsdesign findet eine Konzentration auf eine oder wenige unabhängige Variablen statt. Bei einem Y-zentrierten Forschungsansatz dominiert die „Vergangenheitsperspektive“ das Erkenntnisinteresse: Was erklärt die Varianz der Y-Variablen, also die abhängige Variable? Zentral ist demnach die Einbeziehung möglichst aller theoretisch relevanten erklärenden Variablen, denen ein kausaler Effekt zugeschrieben werden kann.

Der Logik der X- und Y-zentrierten Forschung folgend, wäre die Szenariotechnik als spezifische Methode der X-Zentrierung einzustufen. Die Forschungsliteratur zu dieser Technik ist breit und interdisziplinär angelegt, mit unterschiedlichen Definitionsansätzen. Konsens besteht über folgende Eigenschaften: Erstens hat eine Szenarioanalyse das Ziel, mehrere (meist zwei bis fünf) mögliche Entwicklungen zu beschrieben. Zweitens wird im Rahmen einer Szenarioanalyse zuerst die Gegenwart genauer betrachtet, etwa in Bezug auf eine Gebietskörperschaft. Drittens sucht man die für die künftige Entwicklung kritischen Punkte, die den weiteren Verlauf stark beeinflussen, besonders hervorzuheben.

1 Scharpf, F. W.: Sozialdemokratische Krisenpolitik in Europa, Frankfurt a.M., 1987; Scharpf, F.W.: Games real actors play: actor-centered institutionalism in policy research, Boulder u.a., 1997.

2 Ganghof, S.: Vergleichen in qualitativer und quantitativer Politikwissenschaft: X-zentrierte versus Yzentrierte Forschungsstrategien, in: Kropp, S./Minkenberg, M. (Hg.): Vergleichen in der Politikwissenschaft, Wiesbaden, 2005, 76-93. 
Uneinigkeit herrscht hingegen über die Methode, zu diesem Zukunftsbild zu gelangen; die Literatur weist sehr unterschiedliche Ansätze auf.

Daneben ist vom Szenario die Prognose zu unterscheiden: „Die Szenariotechnik geht weiter als die Prognose im klassischen Sinn, mit der die Vergangenheit fortgeschrieben wird. Mit Hilfe der Szenariotechnik werden die zukünftigen glaubhaften Entwicklungen auf der Basis quantitativer und qualitativer Daten ganzheitlich aufgezeigt. “3 Dagegen sind Prognosen Vorhersagen, die sich vor allem aus Vergangenheitsdaten, etwa über Regressionsanalysen, herleiten lassen. $\mathrm{Zu}$ den Prognosen zählt auch die Extrapolation von Trends über den Haushaltsoder Konjunkturverlauf, von der Vergangenheit in die Zukunft. Bei den Prognoseverfahren existiert ebenfalls ein breites Feld unterschiedlicher Verfahren. So basieren etwa ,intuitive Prognosen“ auf Expertenurteilen. $\mathrm{Zu}$ den intuitivstrukturierten Prognosen zählen das brainstorming oder die Delphi-Methode. Induktiv-mathematische Methoden verwenden vermehrt Regressionsanalysen oder Trendverfahren wie die Zeitreihenanalyse. Deduktiv-nomologische Prognosen wiederum basieren auf Gesetzmäßigkeiten.

Ein drittes auf die Vorhersage der Zukunft abzielendes Verfahren ist die Planung. In Abgrenzung zu den abschätzenden Verfahren zeichnet sie sich durch die Definition eines (konkreten) Ziels aus. In zeitlicher Hinsicht kann dabei zwischen taktischer Planung (kurz- bis mittelfristig) und strategischer Planung (langfristig mit einem Planungszeitraum von drei bis fünf Jahren) unterschieden werden. Im Bereich der Öffentlichen Finanzen erfährt vor allem die mehrjährige Finanzplanung eine zunehmende Aufmerksamkeit, weil in ihr u.a. ein verbindlicher Schuldenabbauplan dokumentiert wird.

Schließlich verbleiben die Simulationsverfahren, die die Wirklichkeit in Modellen nachbilden und mit Hilfe von Computern mögliche Veränderungen abzuschätzen suchen. Dazu werden die Kernparameter eines realen Systems ,durchgespielt“. Simulationen sind daher „Quasi-Experimente“, in denen ein Modell mit unterschiedlichen Werten durchgerechnet wird, um einen breiten Ergebnisraum zu erhalten.

Im Rahmen dieses Beitrags lauten die Forschungsfragen wie folgt: Wie werden sich die Finanzen des Bundes in den kommenden vier Jahren entwickeln? Und: Kann der Bund 2016 einen (strukturell) ausgeglichenen Haushalt vorlegen? Zur

3 Mietzner, D.: Begriff, Nutzen und Funktionen von Szenarien, in: Mietzner, D.: Strategische Vorausschau und Szenarioanalysen, Wiesbaden, 2009, 95-115. 
Beantwortung werden die vier vorgestellten Verfahren der Zukunftsabschätzung eingesetzt. Der Fokus richtet sich dabei auf die zentralen Verschuldungsindikatoren (Schuldenstand und Defizit bzw. Nettokreditaufnahme) sowie auf die Staatsausgaben. In einem ersten Schritt werden zunächst die über Prognosen, Planungen und Simulationen gewonnenen Ergebnisse vorgestellt (II.). Anschließend wird die Szenariotechnik näher erläutert (III.), bevor im Hauptteil des Beitrags vier unterschiedlichen Szenarien diskutiert werden (IV.).

\section{Prognosen, Planungen und Simulationen zur Haushaltspolitik}

\section{Das Domar-Prognosemodell}

Unter dem Stichwort Domar-Modell ${ }^{4}$ werden seit langem die Grenzen der staatlichen Kreditaufnahme diskutiert. Das Domar-Modell zählt zu den deduktivnomologischen Prognosemodellen, also jenen Verfahren, die auf der Basis von Gesetzmäßigkeiten berechnet werden. Das Domar-Modell verwendet dabei wachstumstheoretische Überlegungen, die sowohl bei der Verschuldung als auch beim Wirtschaftswachstum ansetzen. Langfristig ist aus schuldenarithmetischer Perspektive das Verhältnis von Defizitquote (a) und Wirtschaftswachstum (w) die entscheidende Zielgröße, da sie die Staatsschuldenquote determiniert. ${ }^{5}$ Das Domar-Modell wird in verfeinerter Form auch heute noch zur Abschätzung der künftigen öffentlichen Verschuldung angewendet. Hierbei erlaubt das Modell die Berechnung einer ,natürlichen Grenze“ der Staatsverschuldung, die von anderen (etwa juristischen, politischen oder psychologischen) Grenzen zu unterscheiden ist. Ein zentrales Ergebnis des Modells lautet: Ist der Anteil der Kreditaufnahme am Volkseinkommen größer als das Wirtschaftswachstum, erreicht die Schuldenquote langfristig keine feste Obergrenze. Je stärker beide Größen auseinander fallen (Defizit $>$ Wirtschaftswachstum), desto schneller wird die Schuldenquote ansteigen und desto eher ,explodiert“ die Staatsverschuldung. Werden als (dauerhafte) Defizitquote 3 Prozent, als Wirtschaftswachstum 1 Prozent und als Inflationsrate 2 Prozent angenommen, dann steigt unter Konstanz dieser Parameter die Schuldenquote langfristig auf 100 Prozent des BIP (s. Gleichung 1).

Die neue Schuldengrenze im Grundgesetz lässt ab 2016 nur noch ein Defizit von 0,35 Prozent des BIP zu. Bei einer dauerhaften Inflationsrate von 2 Prozent und

4 Domar, E.D.: The 'Burden of the Debt' and the National Income, in: American Economic Review 34 (1944), $798-827$.

5 Ebd., 824. 
einem Wirtschaftswachstum von 1,0 Prozent würde die Schuldenquote Deutschlands damit auf lange Sicht von aktuell über 80,5 Prozent des BIP auf 11,66 Prozent des BIP sinken. Der Grenzwert der Verschuldung tendiert nun (für $\mathrm{t}$ gegen unendlich), im Modell mit Inflation, gegen das Verhältnis aus Defizitquote sowie der Summe aus Inflationsrate und Wirtschaftswachstum. Je größer die Wachstums- und Inflationsrate, desto geringer fällt die Verschuldungsquote aus. Entscheidend ist vor allem das Wirtschaftswachstum als verschuldungshemmende Determinante, da die Inflation konstant und exogen kontrolliert bleibt.

$$
\text { Gleichung 1: } \quad \operatorname{Lim}_{t \rightarrow \infty} \frac{D}{Y}=\frac{\mathrm{a}}{\mathrm{w}+\mathrm{p}}
$$

Mit: $\quad D=$ nominaler Schuldenstand

$Y=$ nominales Bruttoinlandsprodukt

$\mathrm{a}=$ Haushaltsdefizit / Nettokreditaufnahme (Defizitquote)

$\mathrm{W}=$ jährliches Wirtschaftswachstum

$\mathrm{p}=$ jährliche Inflationsrate

Dies bedeutet in letzter Konsequenz auch: Wirtschaftswachstum ist eine Notwendigkeit zur Konsolidierung der öffentlichen Haushalte - solange man keine massiven Einschnitte plant.

In Tab. 1 werden die Ergebnisse von vier unterschiedlichen Prognosen mit variierenden Annahmen summiert. Dabei ist allen Prognosen gleich, dass von einer stets konstanten Inflationsrate von 2 Prozent ausgegangen wird. Im ersten Modell wird vermutet, dass die gesamtgesellschaftliche Defizitquote auf dem für den Bund ab 2016 zulässigen Wert von 0,35 Prozent des BIP verharrt. Bei einem angenommenen Wirtschaftswachstum von einem Prozent würde dies eine langsame und stetige Schuldenquotenreduktion bedeuten. Wird der Referenzwert des Vertrages von Maastricht mit einem zulässigen Defizit von 3 Prozent des BIP zugrunde gelegt (vgl. Projektion 2 in Tab. 1), dann steigt die Schuldenquote langsam an. Die dritte Projektion basiert auf den Annahmen der Regierung für die kommenden Jahre. Darin geht die Bundesregierung von einem Wirtschaftswachstum von 1,6 Prozent für die kommenden Jahre aus, die Kreditaufnahme des öffentlichen Gesamthaushalts liegt in dem Bericht bei etwa 0,1 Prozent des BIP. ${ }^{6}$ Diese ,optimistische“ Variante würde die Verschuldungslast spürbar reduzieren. Die Schuldenstandsquote sänke bei einer Konstanz der getroffenen An- 
nahmen in der nächsten Legislaturperiode um rund 10 Prozentpunkte. Mittelfristig würde sich das Problem deutlich entschärfen.

Tabelle 1: Prognose des Schuldenstandes auf Basis des Domar-Modells

\begin{tabular}{c|c|c|c|c}
\hline & $\begin{array}{c}\text { Projektion 1 } \\
\text { „Einhaltung der } \\
\text { Schuldenregel“ }\end{array}$ & $\begin{array}{c}\text { Projektion 2 } \\
\text { „Maastricht- } \\
\text { Szenario“ }\end{array}$ & $\begin{array}{c}\text { Projektion 3 } \\
\text { "Projektion der } \\
\text { Regierung“ }\end{array}$ & $\begin{array}{c}\text { Projektion 4 } \\
\text { "worst case“ }\end{array}$ \\
\hline \multirow{2}{*}{ Annahmen: } & $\mathrm{a}=0,0035$ & $\mathrm{a}=0,03$ & $\mathrm{a}=0,001$ & $\mathrm{a}=0,04$ \\
& $\mathrm{w}=0,01$ & $\mathrm{w}=0,01$ & $\mathrm{w}=0,016$ & $\mathrm{w}=0,005$ \\
$\mathrm{p}=0,02$ & $\mathrm{p}=0,02$ & $\mathrm{p}=0,02$ & $\mathrm{p}=0,02$ \\
\hline $\mathbf{2 0 1 4}$ & 78,47 & 81,08 & 77,75 & 82,46 \\
$\mathbf{2 0 1 5}$ & 76,49 & 81,64 & 75,10 & 84,38 \\
$\mathbf{2 0 1 6}$ & 74,58 & 82,18 & 72,54 & 86,24 \\
$\mathbf{2 0 1 7}$ & 72,72 & 82,71 & 70,08 & 88,07 \\
\hline $\mathbf{2 0 2 0}$ & 67,46 & 84,19 & 63,19 & 93,26 \\
$\mathbf{2 0 2 5}$ & 59,69 & 86,40 & 53,24 & 101,10 \\
\hline
\end{tabular}

Anmerkung: Berechnungen auf Basis der Gleichung 1. Dargestellt sind die Verschuldungsquoten in Prozent des BIP.

Die vierte Prognose kann als worst case-Projektion verstanden werden, die sich einstellen dürfte, wenn eine Verschärfung der Euro- und Schuldenkrise eintritt. Die Annahme eines Defizits in Höhe von 4 Prozent des BIP wäre nicht unrealistisch und auch nur ein schwaches Wachstum von 0,5 Prozent könnte in diesem Fall als plausibel angesehen werden. Die Konsequenzen wären weiter steigende Schuldenquoten und ein mittelfristiges Erreichen der 100 Prozent-Marke.

Welche der Prognosen am wahrscheinlichsten ist, hängt sowohl von der globalen als auch von der Entwicklung im Euro-Raum ab. So sind steigende Inflationsraten angesichts hoher „Target-Salden“ und der Staatsschuldenfinanzierung in Südeuropa wahrscheinlicher als noch vor einigen Jahren. Möglicherweise wird die EZB auch auf eine restriktivere Geldpolitik umschwenken, was sich eher negativ auf das Wachstum auswirken würde.

Zusammenfassend kann festgestellt werden, dass selbst bei einer kleinen Variation der Annahmen des Domar-Modells der outcome schon kurz- und mittelfristig stark variiert: So sind bereits nach vier Jahren 25 Prozentpunkte Differenz zwischen der schlechtesten und der besten Prognose zu verzeichnen; nach 12 Jahren liegt diese bei 90 Prozentpunkten. Das Defizit bzw. die Nettokreditaufnahme ist der am ehesten von der Politik steuerbare Faktor, obwohl auch diese Größe stark 
von exogenen Faktoren, wie der demographischen Entwicklung und dem Wirtschaftswachstum, beeinflusst wird. ${ }^{7}$

Als problematisch sind beim Domar-Modell vor allem zwei Eigenschaften anzusehen: Erstens, die ausgewiesene (explizite) Staatsschuld ist nur die ,halbe Wahrheit", denn sie erfasst nicht, was die nachfolgenden Generationen an Belastungen aus heutigen Zahlungsversprechen zu tragen haben (etwa mit Blick auf Pensionsverpflichtungen). Neben der expliziten ist demnach auch die Berechnung der impliziten Staatsverschuldung (über so genannte, intergenerationale Modelle“) von großer Bedeutung. Zweitens geht das Modell von dauerhaft konstanten Werten aus, was der Realität nur wenig entspricht. Durch die Verwendung langfristiger Durchschnitte könnte dieser Fehler etwas ausgeglichen werden. Jedoch fällt die Sensitivität mit aktuelleren Durchschnittswerten größer aus, was die Prognose positiv bzw. negativ beeinflussen kann. ${ }^{8}$

\section{Die Entwicklung auf Basis der mittelfristigen Finanzplanung}

Um die Entwicklung der Staatsfinanzen über einen längeren Zeitraum im Voraus planen zu können, ist die Exekutive sowohl auf Bundes- als auch auf Landesebene verpflichtet, das jährliche Haushaltsgesetz und den Haushaltsplan im Rahmen des Haushaltsaufstellungsverfahrens durch eine mittelfristige Finanzplanung zu ergänzen, die Angaben zur künftigen Ausgaben- und Einnahmenentwicklung enthält. Die mittelfristige Finanzplanung (fünfjährig) ist seit 1967 durch das Stabilitäts- und Wachstumsgesetz (StWG) für den Bund vorgeschrieben, seit 1974/75 für die Länder. War die Finanzplanung in der Vergangenheit ein eher unverbindliches Instrument, so nutzen inzwischen einige Bundesländer das Instrument als strategische Ressource in einem „Delegations-Ansatz“ zur Haushaltssteuerung und Haushaltskonsolidierung. ${ }^{9}$ In einer Befragung der 16 Haushaltsverantwortlichen ergab sich ein deutlicher Bedeutungszuwachs. So wird die mittelfristige Finanzplanung von 2/3 aller Befragen als nützliches Instrument zur Durchsetzung politischer Ziele angesehen, wodurch ihr eine gewisse Bedeutung im politischen Prozess zukommt. ${ }^{10}$ Eher unzureichend fällt dagegen die nach-

7 Wagschal, U.: Staatsverschuldung: Ursachen im internationalen Vergleich, Opladen, 1996.

8 Blaas, W./Matzner, E.: Nachlassendes wirtschaftliches Wachstum und Staatsverschuldung, in: Simmert, D. B./Blaas, W. (Hg.): Staatsverschuldung kontrovers, Köln, 1981, 118-136.

9 Vgl. Wagschal, U./Wenzelburger, G./Metz, T./Jäkel, T.: Konsolidierungsstrategien der Bundesländer: Verantwortung für die Zukunft, Gütersloh, 2009, 290ff.

10 Ebd. 
trägliche Kontrolle der in der mittelfristigen Finanzplanung gemachten Vorgaben bzw. Projektionen aus, die nur in der Hälfte der Bundesländer stattfindet.

Der Finanzplan, der jedes Jahr neu aufgestellt wird, unterliegt einem festen Rhythmus: die mittelfristige Planung läuft ,rollierend“ über die fünf Jahre. Dieser gleitende Finanzplan wird somit jedes Jahr neu aufgestellt und um ein Jahr fortgeschrieben. Damit kann flexibler auf Veränderungen der ökonomischen Rahmenparameter reagiert werden. Das erste Jahr ist das laufende Haushaltsjahr, das zweite Jahr wird durch den Haushaltsentwurf des kommenden Jahres abgedeckt. Anschließend folgen somit drei ,echte“ Planungsjahre. Für die Auswertung wird dann das letzte Planungsjahr verwendet und mit den Ist-Zahlen verglichen. Kern der Planungsevaluation ist somit die Frage, wie die Prognosen des Bundes im Vergleich zur ex-post-Realität aussehen. Hierzu wurde für den Zeitraum von 1999 bis 2013 die mittelfristige Finanzplanung mit den tatsächlichen Ist-Ergebnissen verglichen. In Tab. 2 werden dazu zunächst die Nettokreditaufnahmen und der angestrebte Termin des Haushaltsausgleichs zum Vergleich herangezogen.

Tabelle 2: Soll-Ist-Vergleich der Nettokreditaufnahme (NKR) in der Mittelfristigen Finanzplanung

\begin{tabular}{c|c|c|c|c|c}
\hline $\begin{array}{c}\text { Finanzplan } \\
\text { des Jahres } \\
(\boldsymbol{t})\end{array}$ & $\begin{array}{c}\text { Bezugsjahr } \\
(\boldsymbol{t}+\mathbf{5})\end{array}$ & $\begin{array}{c}\mathbf{( 1 )} \\
\text { Geplante } \\
\text { NKR in t+5 }\end{array}$ & $\begin{array}{c}\mathbf{( 2 )} \\
\text { Ist-NKR } \\
\text { in t+5 }\end{array}$ & $\begin{array}{c}\text { Differenz } \\
\mathbf{( 1 )}-\mathbf{( 2 )}\end{array}$ & $\begin{array}{c}\text { Haushalts- } \\
\text { ausgleich } \\
\text { geplant für }\end{array}$ \\
\hline 1999 & 2003 & 15,5 & 38,6 & $-23,1$ & 2006 \\
\hline 2000 & 2004 & 10,2 & 39,5 & $-29,3$ & 2006 \\
\hline 2001 & 2005 & 5,0 & 31,2 & $-26,2$ & 2006 \\
\hline 2002 & 2006 & 0,0 & 27,9 & $-27,9$ & 2006 \\
\hline 2003 & 2007 & 10,0 & 14,3 & $-4,3$ & $k \cdot A \cdot$ \\
\hline 2004 & 2008 & 19,5 & 11,5 & 8,0 & $k \cdot A \cdot$ \\
\hline 2005 & 2009 & 20,0 & 34,1 & $-14,1$ & $k \cdot A \cdot$ \\
\hline 2006 & 2010 & 20,5 & 44,0 & $-23,5$ & $k \cdot A \cdot$ \\
\hline 2007 & 2011 & 0,0 & 17,3 & $-17,3$ & 2011 \\
\hline 2008 & 2012 & 0,0 & 22,5 & $-22,5$ & 2011 \\
\hline 2009 & 2013 & 45,9 & 25,0 & 20,9 & 2016 \\
\hline 2010 & 2014 & 24,1 & ${ }^{a} 6,2$ & 17,9 & 2016 \\
\hline 2011 & 2015 & 14,7 & $n \cdot a$. & - & 2016 \\
\hline 2012 & 2016 & 0,00 & $n \cdot a \cdot$ & - & 2016 \\
\hline 2013 & 2017 & 0,00 & $n \cdot a \cdot$ & - & 2016 \\
\hline $\begin{array}{c}\text { Durchschnitt } \\
\text { (FP 1999-2010) }\end{array}$ & & 14,2 & 26,0 & $-11,8$ & \\
\hline
\end{tabular}

Anmerkungen: Alle Werte in Mrd. Euro, d.h. Werte in DM wurden zum amtlichen Wechselkurs in Euro umgerechnet (1,95583 DM/EUR); a = Entwurf im Bundeshaushalt für 2014, k.A. = keine Angabe im jeweiligen Finanzplan; n.a. = nicht angegeben; NKR $=$ Nettokreditaufnahme, Quellen: Finanzpläne des Bundes seit 1999. 
Daraus ergibt sich eine Reihe von Befunden: In keinem Jahr wurde die aufgestellte Prognose tatsächlich auch nur annähernd erreicht, selbst wenn man eine Fehlermarge von plus/minus 10 Prozent für den Planungszeitraum zulässt. Die Finanzplanung ist somit im Vergleich zum tatsächlichen Haushaltsergebnis stets zu optimistisch. Im Durchschnitt lagen die Planungswerte um 11,8 Milliarden Euro über den Ist-Werten, wobei unter den zwölf vorliegenden Vergleichswerten nur drei Mal weniger Kredite aufgenommen wurden als geplant. Die Finanzplanung für die aktuelle Legislaturperiode kann allerdings nur für die beiden ersten Jahre (2009 und 2010) seriös evaluiert werden. In beiden Jahren fiel die Nettokreditaufnahme höher als erwartet aus, was u.a. an den Kosten der Euro- und Schuldenkrise lag. Zudem fällt auf, dass die im Jahr 2009 geplanten Ausgaben für das Jahr 2013 von 313,5 in der Realität auf 302 Milliarden Euro sanken. Wesentliche Ursache hierfür war die Zinsdividende der Eurokrise, die es Deutschland ermöglichte, sich weit günstiger als geplant zu verschulden. Besonders deutlich wird dieser Effekt, wenn man die geplanten Zinsausgaben (Soll und Ist) für 2013 vergleicht. Im Finanzplan 2009 wurden für 2013 insgesamt 52,07 Milliarden Euro an Zinsausgaben geplant, die sich im aktuellen Haushaltsplan auf 31,63 reduzierten. Vor dem Hintergrund der „eingesparten“ 20,44 Milliarden Euro an Zinsen stellt sich der Ausgabenrückgang von 11,5 Milliarden Euro weniger beeindruckend dar. Rein saldentechnisch sind diese nicht verausgabten Zinsen tatsächlich ein wesentlicher Grund für die um 20,9 Milliarden abgesenkte Nettokreditaufnahme in 2013. Im Ergebnis hat jeder Finanzminister seit 1999 das Ziel des Haushaltsausgleichs anvisiert - soweit absehbar - verfehlt. Der Lackmustest für die gegenwärtige Bundesregierung steht allerdings erst 2016 an. Die dürftige Performanz der mittelfristigen Finanzplanung ist im Übrigen auch der Methodik geschuldet. So wird die konjunkturelle Entwicklung nur für die beiden ersten Jahre berücksichtigt. Für die anschließende mittelfristige Zielprojektion der nächsten drei Planungsjahre werden bewusst keine konjunkturellen Schwankungen mit einbezogen. ${ }^{11}$

Auf Basis der Erfahrungen (und Daten) der vergangenen Jahre kann zudem eine Prägewirkung der durch die Föderalismusreform II eingeführten Schuldengrenze festgestellt werden. Seit 2009 sind die Planungswerte rückläufig und auch die Varianz des Zielerreichungsgrads fiel geringer aus. Insofern scheint die striktere Verschuldungsgrenze einen größeren Effekt zu zeitigen als die alte ,goldene Regel“" des Artikel 115 GG a.F. 
Tabelle 3: Soll-Ist-Vergleich der Ausgaben und Einnahmen in der Mittelfristigen Finanzplanung

\begin{tabular}{c|c|c|c|c|c}
\hline $\begin{array}{c}\text { Finanzplan } \\
\text { des Jahres } \\
(\boldsymbol{t})\end{array}$ & $\begin{array}{c}\text { Bezugsjahr } \\
(\boldsymbol{t}+\mathbf{5})\end{array}$ & $\begin{array}{c}\text { Geplante } \\
\text { Ausgaben in } \\
\boldsymbol{t + 5}\end{array}$ & $\begin{array}{c}\text { Ist- } \\
\text { Ausgaben in } \\
\boldsymbol{t + 5}\end{array}$ & $\begin{array}{c}\text { Geplante } \\
\text { Einnahmen } \\
\text { in } \boldsymbol{t + 5}\end{array}$ & $\begin{array}{c}\text { Ist- } \\
\text { Einnahmen } \\
\text { in } \boldsymbol{t + 5}\end{array}$ \\
\hline 1999 & 2003 & 257,6 & 256,7 & 242,0 & 218,5 \\
\hline 2000 & 2004 & 256,9 & 251,6 & 246,7 & 212,1 \\
\hline 2001 & 2005 & 254,4 & 259,8 & 249,4 & 228,6 \\
\hline 2002 & 2006 & 249,4 & 261,0 & 240,5 & 233,1 \\
\hline 2003 & 2007 & 254,9 & 270,4 & 244,9 & 256,1 \\
\hline 2004 & 2008 & 260,0 & 282,3 & 240,5 & 270,8 \\
\hline 2005 & 2009 & 275,3 & 292,3 & 255,3 & 258,2 \\
\hline 2006 & 2010 & 276,8 & 303,7 & 256,3 & 259,7 \\
\hline 2007 & 2011 & 289,7 & 296,3 & 289,7 & 278,9 \\
\hline 2008 & 2012 & 300,6 & 306,8 & 300,6 & 284,0 \\
\hline 2009 & 2013 & 313,5 & $310,0^{\text {a }}$ & 267,6 & $284,5^{\text {a }}$ \\
\hline 2010 & 2014 & 301,1 & $295,5^{\text {a }}$ & 277,0 & $289,0^{\text {a }}$ \\
\hline 2011 & 2015 & 315,0 & $n \cdot a$. & 300,3 & $n . a$. \\
\hline 2012 & 2016 & 309,9 & $n \cdot a$. & 309,9 & $n . a$. \\
\hline 2013 & 2017 & 317,7 & $n \cdot a$. & 317,7 & $n . a$. \\
\hline
\end{tabular}

Anmerkungen: Alle Werte in Mrd. Euro, d.h. DM-Werte wurden umgerechnet (vgl. unter Tab. 1); a = Entwicklung der öffentlichen Haushalte bis 2017, Übersicht des Bundesfinanzministeriums vom Juli 2013; $n$. $a .=$ nicht angegeben. Berechnet man die Differenz der Ist-Einnahmen und der Ist-Ausgaben, dann ergeben sich für die Jahre 2012-2014 kleine Unterschiede zu den Werten in Tab. 2 und der IstNettokreditaufnahme. Dies liegt an der Verwendung aktueller Zahlen. Quellen: Finanzpläne des Bundes seit 1999 und Entwicklung der öffentlichen Haushalte bis 2017.

Aus den Finanzplanungsdaten können weitere Ursachen für den Konsolidierungserfolg der vergangenen Jahre herausgefiltert werden. So ließen die vergleichsweise gute Wirtschaftslage und die konservativen Einnahmeschätzungen die Ist-Steuereinnahmen über Jahre hinweg höher als erwartet ausfallen (vgl. Tab. 3), insbesondere ab 2007. Diese positiven Überraschungen, sog. windfall profits, waren gerade auch im internationalen Vergleich für erfolgreiche Konsolidierungsanstrengungen verantwortlich. ${ }^{12}$ Auf der Ausgabenseite können ebenfalls verschiedene positiv wirkende Faktoren beobachtet werden. Seit 2010 wur-

12 Wagschal, U./Wenzelburger, G.: Haushaltskonsolidierung, Wiesbaden, 2008. 
de weniger verausgabt als noch fünf Jahre zuvor veranschlagt. Auffallend ist die Konstanz der Ausgaben. So sind die Aufwendungen für die Arbeitsmarktpolitik deutlich geringer ausgefallen als budgetiert. Diese reflektieren die vergleichsweise gute ökonomische Entwicklung und den starken Rückgang der Arbeitslosigkeit nach den Hartz-Reformen. Unerwartet stärkere Ausgabensteigerungen fanden sich zwar auch, etwa bei Rentenleistungen, familienpolitische Leistungen und im Hochschulbereich, doch hielten sich diese noch in Grenzen. Im internationalen Vergleich haben manche Länder, wie etwa Schweden, durch die Reform des Budgetprozesses und die Stärkung der mittelfristigen Finanzplanung die Ausgabenobergrenzen bereits für die drei folgenden Jahre verbindlich festgelegt, so dass das Instrument „Finanzplanung“ als wichtige strategische Ressource betrachtet werden kann. ${ }^{13}$

\section{Simulationen auf der Basis von Regressionsanalysen}

Eine weitere Möglichkeit des Abschätzens künftiger Entwicklungstendenzen wäre die Anwendung von Simulationen auf der Basis von Regressionsanalysen. So zeigen ökonometrische Untersuchungen zu den Auswirkungen von Bankenund Finanzkrisen, dass auch wirtschaftliche Schocks auf die relevanten Verschuldungsgrößen einwirken. ${ }^{14}$ Daneben sind in der Simulationsforschung spezialisiertere Techniken zur Abschätzung künftiger Entwicklungen erkennbar. ${ }^{15}$ Der Vorteil der Regressionsanalyse liegt jedoch in ihrem Bezug zu theoretisch fundierten Kausalmodellen. Zur Modellierung der Determinanten von Staatsverschuldung liegt eine Vielzahl entsprechender Studien vor. In der empirischen Analyse zeigt sich, dass vor allem sozioökonomischen Faktoren Relevanz zukommt, wie etwa der Arbeitslosenquote, dem Wirtschaftswachstum und dem Leistungsbilanzsaldo. Auch politisch-institutionelle Variablen können einen Beitrag zur Erklärung leisten. ${ }^{16}$ Der Ertrag solcher Regressionsanalysen variiert jedoch immer mit einer Reihe von Grundannahmen: Zunächst sind die Ergebnisse von der Fallauswahl abhängig. Je heterogener die untersuchten Fälle, desto größer fällt in der Tendenz die Variation der Ergebnisse aus. Auch sind Querschnittsvergleiche anders zu interpretieren als Längsschnittvergleiche: Bei Ver-

13 Joumard, I./Kongsrud, P.M./Nam, Y.S./Price, R.: Enhancing the Effectiveness of Public Spending in OECD Countries, in: OECD Economics Department Working Papers, 380 (2004).

14 Reinhart, C.M./Rogoff, K.S.: This Time Is Different: A Panoramic View of Eight Centuries of Financial Crises, Princeton, 2009, 249.

15 Gilbert, N./Troitzsch, K.G.: Simulation for the Social Scientist, 2nd ed., New York, 2005.

16 Vgl. Wagschal, U., a.a.O., 1996. 
gleichen für nur ein Land entlang einer Zeitachse erweisen sich die Kontext- und Rahmenbedingungen als stabiler. Auch spielt eine Rolle, welcher Untersuchungszeitraum betrachtet wird: Längere Zeiträume können andere Ergebnisse hervorbringen als die alleinige Betrachtung der aktuellen Situation. Gesamthaft betrachtet sind die Befunde von Regressionsanalysen, die sich mit den Einflussfaktoren auf die Entwicklung staatlicher Verschuldung befassen, somit nur in Grenzen zum Zweck der Abschätzung künftiger Entwicklungen heranzuziehen. Zugleich können die Befunde aber als plausible Kausalmechanismen für die nachfolgend zu diskutierende Szenariotechnik verwendet werden. ${ }^{17}$

\section{Szenariotechnik}

Eine vierte Methode, künftige Entwicklungen abzuschätzen, stellt die Szenariotechnik dar. Am bekanntesten dürften die eher negativen Szenarien des Club of Rome zur Entwicklung der Welt aus dem Jahr 1972 sein, die zwar überwiegend nicht eintrafen, jedoch bis heute einen Meilenstein der Nachhaltigkeitsforschung darstellen. ${ }^{18}$ Gesamthaft betrachtet war dieser Bericht zu den „Grenzen des Wachstums“ ein Durchbruch der auf Computersimulationen basierenden Szenario-Methode. Heutzutage erfreut sich diese Vorgehensweise in der Praxis (u.a. beim Militär) wie auch in unterschiedlichen wissenschaftlichen Disziplinen (hier v.a. in der Ökonomie) einer wachsenden Beliebtheit. ${ }^{19}$ Für die Sozialwissenschaften liegt der Vorteil der Methode in einem „Denken in Zusammenhängen“, also einer mehrdimensionalen Argumentation, sowie der Diskussion und Entwicklung alternativer Denkansätze. Methodisch sind Szenarien auch deshalb interessant, weil sie die Zukunft unter Berücksichtigung unterschiedlicher quantitativer und qualitativer Einflussfaktoren abschätzen.

"A scenario is a policy analysis tool that describes a possible set of future conditions. The most useful scenarios (for corporations, for policy decision makers) are those that display the conditions of important variables over time. In this approach, the quantitative underpinning enriches the narrative evolution of conditions or evolution of the variables; narratives describe the important events and developments that

17 Stellvertretend für viele: Woo, J.: Economic, political, and institutional determinants of public deficits, in: Journal of Public Economics, 87 (2003), 387-426; Franzese, R.: Macroeconomic policies of developed democracies, Cambridge u.a., 2002.

18 Meadows, D.H./Meadows, D.L./Randers, J./Behrens, W.: The Limits to Growth, New York, 1972.

19 Auch die Vereinten Nationen arbeiten mit der Szenariotechnik und gehen in ihrem aktuellen Bericht für 2013 von drei unterschiedlichen Wachstumsszenarien für die Weltwirtschaft aus. Vgl. United Nations: World Economic Situation and Prospects 2013, New York, 2012. 
shape the variables. In terms of innovative methods for policy analysis, the foresight and scenario building methods can be an interesting reference for the social sciences. $" 20$

Allerdings stellt ein Szenario kein umfassendes Bild der Zukunft dar, da seine Funktion eher darin besteht, die Wahrnehmung gezielt auf einen oder mehrere bestimmte, abgegrenzte Ausschnitte der Wirklichkeit zu lenken. ${ }^{21}$ Die zentralen Eigenschaften von Szenarien sind: ${ }^{22}$

- die Entwicklung alternativer Zukunftsbilder, ausgehend von der Gegenwart, anstelle der Weiterentwicklung vergangener Trends;

- die Berücksichtigung sowohl qualitativer Perspektiven als auch quantitativer Daten;

- die Berücksichtigung einschneidender Störereignisse sowie

- das Hinterfragen strategischer Basisannahmen.

Typischerweise entwickelt man neben einem positiven und negativen Extremszenario (die jeweils die günstigste bzw. ungünstigste Entwicklung darstellen) noch ein sog. Trendszenario, in dem die heutige Situation in die Zukunft fortgeschrieben wird. ${ }^{23}$ Diese Szenarien bilden einen sog. Szenario-Trichter, der die möglichen outcomes umfasst. Daneben sind zusätzliche Alternativszenarios üblich, etwa um bestimmte Einflussgrößen genauer herauszuarbeiten. Weiterhin kann man mit Blick auf etwaige Zielvorgaben zwischen Idealszenarien und Realszenarien differenzieren. Erstere sind präskriptiver und normativer Natur, während letztere auf der Basis möglichst vieler Daten und Fakten entwickelt werden. Schließlich existiert eine Vielzahl von Methoden, die in der Szenario-Analyse Anwendung finden, darunter die Delphi-Technik, Entscheidungsbäume, morphologische Analyse u.a.m. ${ }^{24}$

Typischerweise orientiert sich das Verfahren einer Szenarioanalyse an aufeinanderfolgenden Phasen (etwa: Problemfeldanalyse, Einflussanalyse, Trendprojektion und Szenarienermittlung, Bewertung). Dies wird im Folgenden anhand von

20 Brandão Moniz, A.: Scenario-Building Methods as a Tool for Policy Analysis, in: Rihoux, B./Grimm, H. (ed.): Innovative Comparative Methods for Policy Analysis, New York, 1986.

21 Kosow, H./Gaßner, R.: Methoden der Zukunfts- und Szenarioanalyse. Überblick, Bewertung und Auswahlkriterien, Berlin, 2008, 10.

22 Mietzner, D.: Strategische Vorausschau und Szenarioanalyse: Methodenevaluation und neue Ansätze, Wiesbaden, 2009, 99.

23 Fahey, L./Randall, R.M.: What is Scenario Learning, in Fahey, L./Randall, R.M. (ed.): Learning from the Future: Competitive Foresight Scenarios, New York, 1998, 6.

24 Kosow, H./Gaßner, R.: a.a.O. 
vier Szenarien durchgespielt: Dem best- und worst-case-Szenario, einem Trendszenario ohne politische Eingriffe und ohne Koordinierung sowie einem Trendszenario mit politischen Eingriffen und verstärkter Koordinierung.

\section{Vier Szenarien - die Entwicklung der Verschuldung des Bundes}

\section{Ausgangslage und Problemfeldanalyse}

Nach der Föderalismusreform II im Jahr 2009 und der damit verbundenen Einführung einer grundgesetzlichen Verschuldungsgrenze darf das Defizit des Bundeshaushalts ab 2016 nur noch max. 0,35 Prozent des Bruttoinlandproduktes betragen. Diese Schuldenbremse, die in Anlehnung an eine ähnliche Regelung in der Schweizer Bundesverfassung eingeführt wurde, schafft einen deutlichen Handlungsdruck für die Bundesregierung, sofern diese ihre Glaubwürdigkeit angesichts der wiederholten Ankündigung von Schuldenabbauprogrammen erhalten möchte. Die Einführung dieser Schuldenregel, die gegenüber der zuvor geltenden ,goldenen Regel“ (derzufolge das Defizit lediglich kleiner als die Investitionen ausfallen musste) eine deutliche Verschärfung vorsieht, ist vor dem Hintergrund des Verschuldungsanstiegs der vergangenen 50 Jahre zu sehen.

In der frühen Bundesrepublik Deutschland der 1950er Jahre war man aufgrund von Handelsüberschüssen schnell in der Lage, Devisenreserven aufzubauen und damit die interne wie externe Verschuldung abzubauen. Es gelang sogar, Budgetüberschüsse zu erzielen. Unter dem Finanzminister Schäffer häufte der Bund in hohem Maß liquide Mittel an, die unter der Bezeichnung „Julius-Turm“ in die Geschichte eingingen. So blieb die Staatsverschuldung im Zeitraum von 1950 bis 1973 gering und konstant. Die Schuldenquote betrug mit rund 20 Prozent des BIP nur rund ein Viertel des heutigen Niveaus. Selbst zu Beginn der Ölpreiskrise 1973, dem Auslöser für den ersten Verschuldungsschub, lag sie noch bei lediglich 17,8 Prozent des BIP.

Der Anstieg der Verschuldung erfolgte nicht kontinuierlich, sondern in Reaktion auf Krisen oder einschneidende Ereignisse: Besonders stark stiegen die Schulden während den Ölpreiskrisen (Zuwächse von je 9 Prozentpunkten zwischen 1973 und 1977 bzw. 1980 und 1984), sodann im Gefolge der Deutschen Einheit (rd. 20 Prozentpunkte zwischen 1991 und 1997), nach dem Platzen der Dot-ComBlase (rd. 9 Prozentpunkte zwischen Zuwachs 2001 und 2005) sowie im Gefolge der Finanz- und Wirtschaftskrise 2007/08 (rd. 18 Prozentpunkte zwischen 2007 und 2010). In der Summe betrug der Verschuldungszuwachs in Krisenzeiten rd. 65 Prozentpunkte des BIP. In den dazwischenliegenden Zeiträumen blieb die 
Verschuldungsquote meist konstant (1984-1990) oder sank sogar (etwa seit 2011).

\section{Einflussfaktoren}

Im Rahmen der sich anschließenden Einflussanalyse (samt Deskriptorenbestimmung) sind alle Faktoren zu identifizieren, die auf das Untersuchungsfeld unmittelbar einwirken. Auf eine Einflussmatrix, die typischerweise erstellt wird und die die wechselseitigen Zusammenhänge unter den Einflussfaktoren bewertet, wird hier jedoch verzichtet. Es werden vielmehr nur direkt wirkende Faktoren betrachtet. Hierbei wird nicht zwischen „,negativen“ und „positiven“ Einflüssen unterschieden, sondern es wird die Stärke des Einflusses auf die abhängigen Variablen berücksichtigt. Als Basis hierfür können theoretische Überlegungen und Zusammenhänge dienen, vor allem aber die empirischen Befunde aus der vorliegenden Forschungsliteratur. Als zentrale Größen (also die zu beeinflussenden Variablen) werden die Verschuldungsdaten (Defizit und Schuldenstand) sowie die Einnahmen und Ausgaben angesehen (vgl. Tab. 5).

Tabelle 4: Schuldenstand und Zinszahlungen des Bundes (in Mrd. EUR)

\begin{tabular}{c|c|c|c}
\hline & $\mathbf{2 0 0 0}$ & $\mathbf{2 0 1 3}$ & $\mathbf{\Delta ~ 2 0 0 0 - \mathbf { 2 0 1 3 }}$ \\
\hline $\begin{array}{c}\text { Nominaler Schul- } \\
\text { denstand }\end{array}$ & 774 & 1.078 & $+39,3 \%$ \\
\hline $\begin{array}{c}\text { Zins- } \\
\text { zahlungen }\end{array}$ & 39,9 & 31,7 & $-21,4 \%$ \\
\hline $\begin{array}{c}\text { Durchschnittliche } \\
\text { Verzinsung }\end{array}$ & $5,1 \%$ & $2,9 \%$ & $-2,2$ Prozentpunkte \\
\hline
\end{tabular}

Quellen: Finanzberichte des Bundes.

Ein maßgeblicher Faktor, der zur positiven Haushaltsentwicklung der vergangenen drei Jahre beitrug, war der Rückgang des Zinsniveaus. Die Zinsdividende, bedingt durch die Einführung des Euro und die „Stabilitätsanleihe“ der Hartwährungsländer, war ein zentraler Faktor für die Haushaltskonsolidierung in vielen Mitgliedstaaten. Jedoch entfiel für diese Länder zugleich auch die Möglichkeit, ihre Schulden durch Inflation zumindest teilweise zu entwerten und durch Abwertungen ihren Produktivitätsnachteil auszugleichen. Für Deutschland fiel die Zinsdividende nach der Einführung der gemeinsamen Währung zunächst gering aus. Erst im Rahmen der Finanz- und Wirtschaftskrise, die sich zur Euro- und 
Staatsschuldenkrise entwickelte, fielen die Nominalzinsen beträchtlich, wie Tab. 4 verdeutlicht. Während die Schulden von 2000 bis 2013 um knapp 40 Prozent stiegen, sanken die Zinsausgaben um 21,4 Prozent; die effektive Durchschnittsverzinsung fiel dabei um insgesamt 2,2 Prozentpunkte. Läge der Zins weiter auf dem Niveau des Jahres 2000, so müsste der Bund etwa 23 Milliarden an zusätzlichen Zinsaufwendungen einkalkulieren - was einen Haushaltsausgleich in weite Ferne rücken ließe. Das Zinsniveau übt mithin einen starken Einfluss auf die Verschuldungsindikatoren aus.

Weitere bedeutsame Einflussfaktoren sind der Wechselkurs und die Entwicklung des Außenhandels. Deutschland hat wie kaum ein zweites Euro-Land von der Abwertung des Euros und der Euro-Schwäche profitiert. Die strukturelle Unterbewertung der Deutschen Mark war für die alte Bundesrepublik in den 1950er und 1960er Jahren ein wichtiger Erfolgsfaktor, der sich angesichts einer Abwertung des Euro von über 20 Prozent gegenüber dem US-Dollar von Mitte 2008 bis 2012 gleichsam wiederholte (vgl. Abb. 1). Damit verbanden sich sowohl ein starker Anstieg der Exporte (seit 2009) als auch ein wachsender Leistungsbilanzüberschuss. Der Erfolg auf dem Arbeitsmarkt ist damit nicht nur strukturellen Ansätzen, wie etwa den Hartz-Reformen, geschuldet, sondern auch der guten Außenhandelsperformanz.

Abbildung 1: Entwicklung des Wechselkurses EUR/USD und des Außenhandels

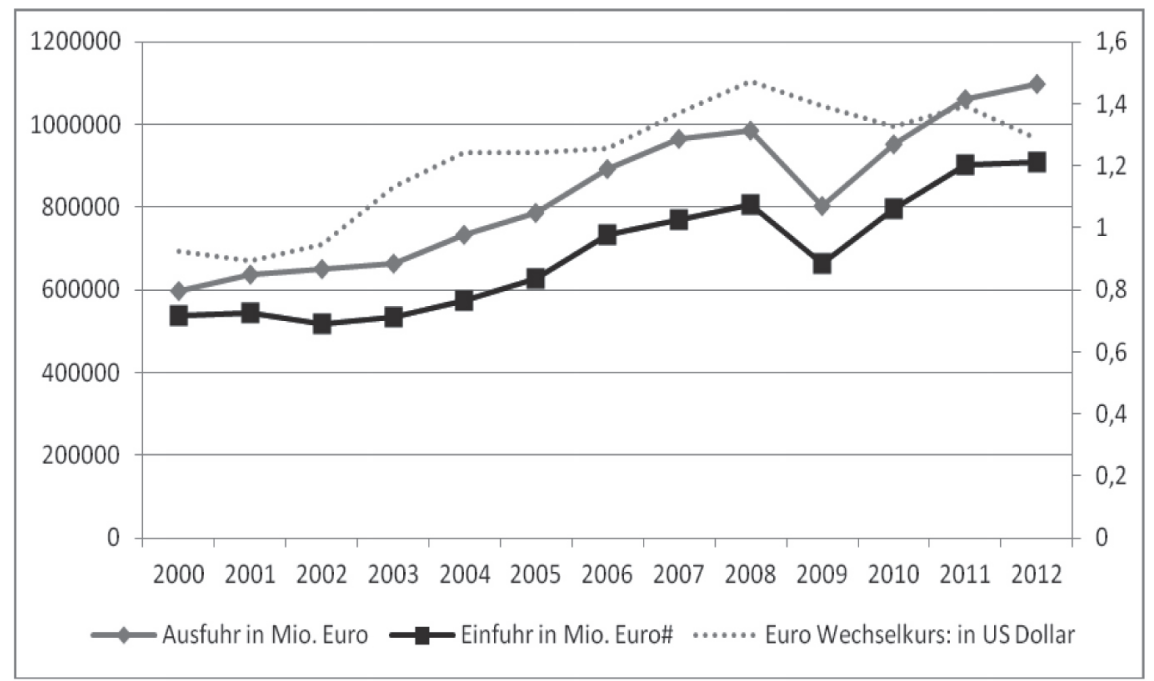

Quellen: Eurostat (Wechselkursdaten), Statistisches Bundesamt (Außenhandelsdaten). 
Fritz Scharpf hat in seinen eingangs zitierten Schriften auf die Bedeutung der makroökonomischen Koordinierung für den wirtschaftspolitischen Erfolg hingewiesen. Eine moderate Lohnpolitik seitens der Gewerkschaften, die durch eine moderate Politik der Notenbank akkommodiert würde, führt zu besseren Ergebnissen als eine expansive Lohnpolitik, die durch eine restriktive Geldpolitik beantwortet wird. ${ }^{25}$ Im Zuge der Euroeinführung wurden solche spieltheoretischen Überlegungen für die Gewerkschaften irrelevant, da eine on-size-fits-allGeldpolitik keine abgestimmten Reaktionen ermöglicht. Hinzu kommt, dass „asymmetrische Schocks“ die Euro-Staaten unterschiedlich treffen, die EZB mithin kaum problemadäquat reagieren kann. Deutschlands Gewerkschaften haben auf die Krisen der 1990er und 2000er Jahre mit einer deutlichen Lohnzurückhaltung reagiert, womit die Wettbewerbsfähigkeit gesteigert wurde. Diese Lohnzurückhaltung wurde durch eine moderat expansive Fiskalpolitik der Regierung begleitet. Allerdings mussten die Gewerkschaften mit der HartzGesetzgebung umfangreiche Strukturreformen hinnehmen, die sie mittragen konnten. So geriet zwar das Prinzip der Koordinierung in eine Krise, die von den eher am Gemeinwohl orientierten Gewerkschaften aber dennoch nicht mit einer

Abbildung 2: Entwicklung der nominalen Lohnstückkosten $(1999=100)$

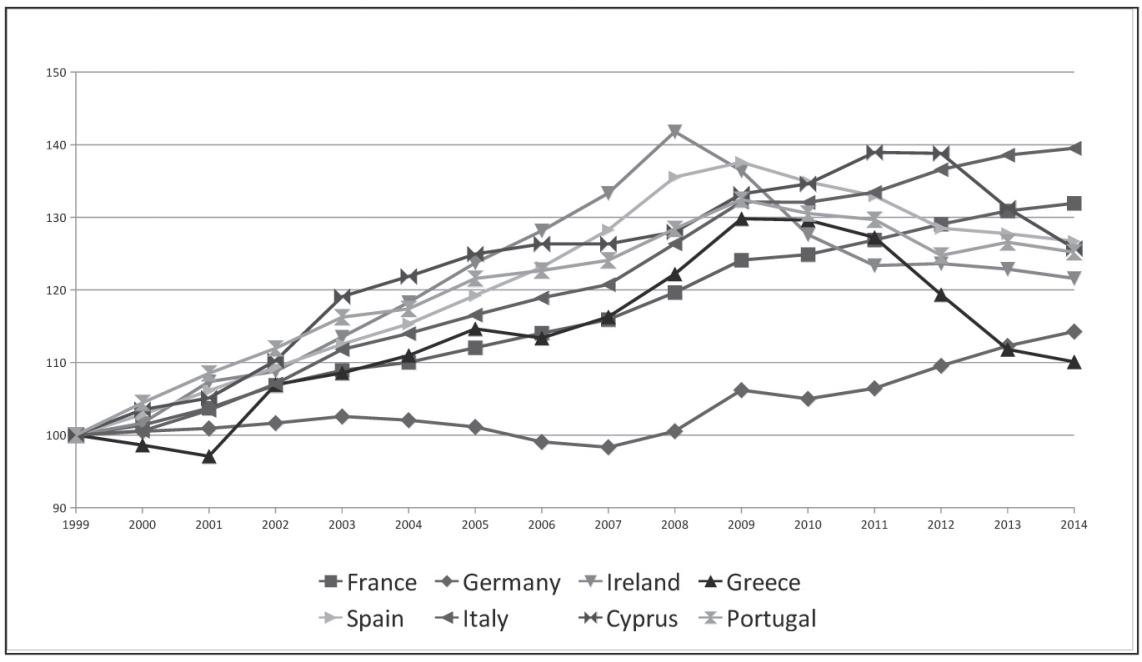

Quelle: Europäische Kommission, ec.europa.eu/economy_finance/ameco.

25 Scharpf, F.: Inflation und Arbeitslosigkeit in Westeuropa. Eine spieltheoretische Interpretation, in: Politische Vierteljahresschrift, 29/1 (1988), 6-41. 
expansiven Strategie beantwortet wurde. ${ }^{26}$ Vor dem Hintergrund der Erfahrungen vergangener Jahrzehnte und sicher auch bedingt durch die Handlungslogik großer sozialer Organisationen ${ }^{27}$ war Lohnzurückhaltung die langfristig bessere Strategie, vor allem im Vergleich zu den expansiven und von Streiks begleiteten Lohnerhöhungen im Süden Europas (vgl. Abb. 2).

Die Faktoren, die sich auf die Staatsverschuldung sowie auf die Einnahmen und Ausgaben auswirken, können generell in exogene und (politisch) endogene Variablen unterschieden werden. Sie werden in Tab. 5 dargestellt, unter Ausweis der Stärke ihres Einflusses (ohne Angabe der Wirkungsrichtung). Gemessen an den empirischen Untersuchungen zur Verschuldung sind vor allem exogene Faktoren für den Verschuldungsanstieg verantwortlich. Exogene Faktoren sind zunächst nicht durch Politiker direkt zu beeinflussen. Neben klassischen Ursachen wie Krieg, Staatszerfall und Systemwechsel sind es vor allem sozioökonomische Faktoren, die zu berücksichtigen sind. Ein starkes Wirtschaftswachstum beispielsweise bremst über mehr Steuereinnahmen und weniger Arbeitslosigkeit den Schuldenanstieg. Und umgekehrt waren makroökonomische Schocks und Krisenereignisse (,displacement effects“) ${ }^{28}$ der Motor für Niveauverschiebungen der Staatsverschuldung nach oben (s.o.). Ein eher moderater Einfluss ist dagegen der Inflation zuzuschreiben. Historisch ist dies zwar eine übliche Variante, Verbindlichkeiten des Staates abzubauen, doch haben dem unabhängige Notenbanken zumeist Grenzen gesetzt. Als exogener Faktor können auch demographische Faktoren gelten. So ist etwa die Seniorenquote eine mächtige Triebkraft der Staatsausgaben und der Staatsverschuldung: Je größer diese Bevölkerungsgruppe ist, desto mehr geben die Regierungen auch für sie aus. Diese Ausgaben können einerseits tatsächlich exogen sein, etwa bedingt durch objektiv höhere Gesundheitsaufwendungen. Andererseits kann dieser demographische Faktor zu einer endogenen Variablen werden, wenn man ihn polit-ökonomisch interpretiert. Als es im Frühjahr 2008 durch die Aussetzung des „Riesterfaktors“ zu einer außerplanmäßigen Rentenerhöhung kam, kommentierte dies Alt-Bundespräsident Herzog in einem Interview der Bild-Zeitung wie folgt: „Ich fürchte, wir sehen gerade die Vorboten einer Rentnerdemokratie: Die Älteren werden immer mehr,

26 Olson, M.: The Logic of Collective Action, Cambridge, 1965.

27 Nach Mancur Olson ist zu erwarten, dass besonders umfassende („encompassing“) Interessengruppen sich am Gemeinwohl orientieren, da sie breite Bevölkerungsgruppen vertreten.

28 Peacock, A./Wiseman, J.: The Growth of Public Expenditure in the United Kingdom, London, 1967. 
Tabelle 5: Einflussfaktoren für zentrale Zielgrößen des Haushalts

\begin{tabular}{|c|c|c|c|c|c|}
\hline \multirow[b]{2}{*}{$\begin{array}{l}\text { Einfluss- } \\
\text { faktor }\end{array}$} & \multicolumn{5}{|c|}{ Zielgrößen des Haushalts } \\
\hline & Ausgaben & Einnahmen & $\begin{array}{c}\text { Kredit- } \\
\text { aufnahme } \\
\text { (kurzfristig) }\end{array}$ & $\begin{array}{l}\text { Schulden- } \\
\text { stand (lang- } \\
\text { fristig) }\end{array}$ & Summe \\
\hline Zinsniveau & 2 & 1 & 2 & 2 & 7 \\
\hline Wechselkurs & 1 & 1 & 1 & 1 & 4 \\
\hline $\begin{array}{l}\text { Wirtschafts- } \\
\text { wachstum }\end{array}$ & 2 & 1 & 2 & 2 & 7 \\
\hline $\begin{array}{l}\text { Arbeits- } \\
\text { losigkeit }\end{array}$ & 2 & 2 & 2 & 1 & 7 \\
\hline $\begin{array}{l}\text { Lohn- } \\
\text { entwicklung }\end{array}$ & 1 & 1 & 0 & 1 & 3 \\
\hline $\begin{array}{c}\text { Inflation \& } \\
\text { Geldpolitik } \\
\text { der EZB }\end{array}$ & 0 & 1 & 1 & 2 & 4 \\
\hline $\begin{array}{l}\text { Demo- } \\
\text { graphische } \\
\text { Entwicklung }\end{array}$ & 1 & 0 & 1 & 2 & 4 \\
\hline $\begin{array}{l}\text { Partei- } \\
\text { politische } \\
\text { Zusammen- } \\
\text { setzung der } \\
\text { Regierung }\end{array}$ & 1 & 1 & 0 & 0 & 2 \\
\hline $\begin{array}{l}\text { Größe der } \\
\text { Regierung }\end{array}$ & 1 & 0 & 0 & 1 & 2 \\
\hline $\begin{array}{l}\text { Schulden- } \\
\text { grenzen }\end{array}$ & 0 & 0 & 1 & 1 & 2 \\
\hline $\begin{array}{l}\text { Bekenntnis } \\
\text { zum } \\
\text { Verschul- } \\
\text { dungsabbau }\end{array}$ & 1 & 0 & 1 & 1 & 3 \\
\hline $\begin{array}{l}\text { Stringenz des } \\
\text { Budget- } \\
\text { prozesses }\end{array}$ & 1 & 0 & 1 & 1 & 3 \\
\hline $\begin{array}{l}\text { Starker } \\
\text { Finanz- } \\
\text { minister }\end{array}$ & 1 & 0 & 1 & 1 & 3 \\
\hline Summe & 14 & 8 & 13 & 16 & 51 \\
\hline
\end{tabular}

Quellen: Finanzberichte des Bundes. 
und alle Parteien nehmen überproportional Rücksicht auf sie. Das könnte am Ende in die Richtung gehen, dass die Älteren die Jüngeren ausplündern. “29

Der in den Literaturbeständen zur Verschuldungsthematik wohl prominenteste endogene Faktor ist die - vermeintlich verschuldungserhöhende - Auswirkung von Wahlen als Teil eines politischen Konjunkturzyklus: Regierungen gleich welcher politischer Couleur neigen vor Wahlen zu verstärkter Schuldenaufnahme. ${ }^{30}$ Da hier v.a. die Zeit nach der kommenden Bundestagswahl betrachtet wird, ist dieser Faktor bestenfalls für die darauf folgende Wahl im Jahr 2017 relevant. In demokratischen Systemen lautet eine der zentralen Fragen im Hinblick auf Varianzen bei der Staatstätigkeit, ob unterschiedliche Parteien zu unterschiedlich starker Verschuldung neigen. In Anlehnung an die Parteiendifferenztheorie von $H_{i b b s}{ }^{31}$ ist davon auszugehen, dass ,linke“ Regierungen eine höhere Verschuldung in Kauf nehmen als ,rechte“, da das vorrangige Ziel der erstbenannten gemäß ihrer Kernwählerschaft - in einer niedrigen Arbeitslosenquote liegt. Zudem sind „linke“ Regierungen eher der Politik des deficit spending zugeneigt, was als ,intellektueller Motor“ für steigende Verschuldungsniveaus angesehen werden kann. Folgt man dagegen der „modifizierten Steuerglättungshypothe$\mathrm{se}^{\text {“ }}{ }^{32}$ so neigen bürgerliche und ,rechte“ Regierungen eher zu verstärkter Schuldenaufnahme, auch hier aufgrund von Erwartungen seitens der Wählerbasis: bürgerliche Regierungen verfolgen demnach das vorrangige Ziel, die Steuerbelastung für ihre Klientel zu vermindern - auch auf Kosten steigender Staatsschulden. Schließlich wird auch Regierungen, die auf Parteienkoalitionen gründen, ein verschuldungserhöhender Effekt zugeschrieben. ${ }^{33}$ Die Begründung liegt in der vermeintlichen Unfähigkeit solcher Regierungen, angesichts unterschiedlichster Sonderinteressen eine glaubwürdige Fiskalpolitik betreiben zu können. De Haan und Sturm konnten diese Vermutung jedoch empirisch nicht bestätigen. $^{34}$

29 „Generationendebatte: Roman Herzog warnt vor Rentnerdemokratie“, online unter www.bild.de.

30 Nordhaus, W.D.: The Political Business Cycle, in: Review of Economic Studies, 42/2 (1975), 169-190.

31 Hibbs, D.A.: Political Parties and Macroeconomic Policy, in: American Political Science Review, 71 (1977), 1467-1487. Hibbs, D.A.: The Partisan Model of Macroeconomic Cycles: More Theory and Evidence for the United States, in: Economics and Politics, 6 (1994), 1-23.

32 Wagschal, U., a.a.0., 1996.

33 Roubini, N./Sachs, J.: Political and Economic Determinants of Budget Deficits in the Industrial Democracies, in: European Economic Review, 33 (1989), 903-933.

34 De Haan, J./Sturm, J-E.: On the relationship between economic freedom and economic growth, in: European Journal of Political Economy, 16/2 (1999), 215-241. 
Spätestens seit Kydland und Prescott wird von Teilen der Wirtschaftswissenschaft die Position vertreten, dass politische Einflussnahme in Demokratien mit Blick auf die Schuldenproblematik entweder ohne Wirkung bleibt oder mehr Schaden als Nutzen anrichtet. ${ }^{35}$ Hinzu kommt das Problem zeitinkonsistenten Verhaltens von Regierungen, die stets versuchen, mehrere Ziele gleichzeitig zu erfüllen (etwa: Abbau der Arbeitslosigkeit und Bekämpfung der Inflation). Übergreifend betonen Kydland und Prescott die Bedeutung einer konsistenten und glaubwürdigen Wirtschaftspolitik mit klaren Regeln. Diese Argumentation stellt eine der wesentlichen intellektuellen Grundlagen für die Einführung von Schuldengrenzen und Schuldenregeln in Demokratien dar. Angesichts der empirischen Erfahrungen und der komparativen Evidenz sind solche Regeln jedoch weit weniger wirkungsvoll als meist angenommen. ${ }^{36}$ So haben entsprechende Obergrenzen weder die jüngsten Finanzkrisen noch die in vielen Staaten erkennbaren Höchststände bei der Verschuldung verhindern können. Länder mit Fiskalregeln verzeichnen zwar einen niedrigeren Verschuldungszuwachs als solche ohne bzw. mit nur schwachen Vorschriften, doch steigt die Verschuldung hier wie dort. Schließlich zeigt sich, dass Fiskalregeln weitgehend ohne Einfluss auf das Ziel der Haushaltskonsolidierung bleiben, also die Rückführung der Staatsverschuldung nicht wirklich vereinfachen. Andere Faktoren erweisen sich hier als wesentlich bedeutsamer, etwa das politische Bekenntnis zum Schuldenabbau, ein stringenter Budgetprozess (top-down-Ansatz) oder ein starker Finanzminister. $^{37}$

Entsprechend fallen die in Tab. 5 dargestellten qualitativen Einstufungen für exogene Faktoren höher aus als für endogenen Variablen. Sozioökonomische Größen, vor allem das Wirtschaftswachstum, die Arbeitslosigkeit und das Zinsniveau, sind damit wichtiger als politisch-institutionelle Faktoren. Die endogenen Faktoren wirken eher langfristig, weshalb auch der Schuldenstand durch langfristige Weichenstellungen beeinflusst werden kann. Der geringste Einfluss ist nach dieser Einschätzung auf die kurzfristigen Einnahmen auszuüben.

35 Kydland, F.E./Prescott, E.C.: Rules Rather than Discretion. The Inconsistency of Optimal Plans, in: The Journal of Political Economy, 85/3 (1977), 473-492.

36 Wagschal, U.: „Allheilmittel oder Budgetmimikry: Wie wirksam sind Verschuldungsgrenzen zur Haushaltskonsolidierung?“, in: Zeitschrift für Zeitschrift für Staats- und Europawissenschaften, 11/3 (2011), 352-382.

37 Hallerberg, M./Strauch, R./ Von Hagen, J.: The Use and Effectiveness of Budgetary Rules and Norms in EU Member States, report prepared for the Dutch Ministry of Finance by the Institute of European Integration Studies, 2001. 


\section{Trendprojektion und Szenarienbildung}

Die künftige Entwicklung der diskutierten Einflussfaktoren unterliegt divergierenden Prognosen, wie ein Blick auf die Zahlen des IWF, der OECD, der EU und des Sachverständigenrats zeigt. Dementsprechend werden die folgenden vier Szenarien diskutiert:

- das positive Extremszenario (best case, Sz. 1),

- das negative Extremszenario (worst case, Sz. 2),

- das Trendszenario a mit nur geringfügigen Eingriffen (negative Variante, Sz. 3) sowie

- das Trendszenario $b$ mit stärkeren Eingriffen und ausgepräger Koordinierung (positive Variante, Sz. 4)

Bei den Berechnungen wird für die Szenarien Sz. 1-3 von einer Inflationsrate von 2 Prozent ausgegangen, während Sz. 4 ein Wert von 3 Prozent zugrundliegt. Die zentralen Parameter für die Bestimmung der Schuldenquote sind somit das Wirtschaftswachstum, die Nettokreditaufnahme sowie die Höhe der Ausgangsverschuldung.

Das positive Extremszenario geht davon aus, dass die angestrebten Verschuldungs- und Haushaltsziele erreicht oder gar übertroffen werden. Der Haushalt wird somit nicht nur strukturell ausgeglichen, sondern es werden ab 2016 auch Überschüsse erzielt, mit denen erstmals seit über 50 Jahren eine Rückführung der Verschuldung erreicht werden kann. Im Jahr 2017 liegt die Schuldenquote demnach wieder bei 60 Prozent des BIP. Eine notwendige Bedingung hierfür ist, dass die Zinsen weiterhin auf niedrigem Niveau gehalten werden. Ebenso muss der Wechselkurs des Euro zum US-Dollar und anderen Währungen weiterhin eher niedrig ausfallen. Die Schwäche des Euro stützt die Exportwirtschaft und wirkt sich positiv auf das Wirtschaftswachstum aus, welches jahresdurchschnittlich generell bei mehr als 1,6 Prozent liegt. Die Euro-Krise und die Staatsschuldenkrisen sucht man durch eine intensivierte Koordinierung unter den europäischen Regierungen in den Griff zu bekommen. Gleichzeitig unternehmen die Krisenländer Strukturreformen und werden durch eine moderate Lohnpolitik wieder wettbewerbsfähig. Die politischen Kräfteverhältnisse im Bund bleiben bestehen, insbesondere wird der Finanzminister noch stärker als bisher auf Ausgabendisziplin achten. Die Bundeskanzlerin erklärt die Haushaltskonsolidierung zur Chefsache und gibt dem Finanzminister (über die Haushaltsordnung) mehr Einspruchsmöglichkeiten gegenüber den Begehrlichkeiten der Fachminister. Der 
Budgetprozess wird stringenter ausgestaltet, Eckwertebeschlüsse und die Finanzplanung werden für ganze Planungsperioden verbindlich vorgeschrieben.

Im negativen Extremszenario hingegen kumulieren sich die Probleme, das Verschuldungsziel wird nicht erreicht. Die Euro-Krise verschärft sich nach der Bundestagswahl 2013, da die Hilfszahlungen für die Schuldenländer nur kurzzeitig Wirkung entfalten. Auch der erste Schuldenschnitt für Griechenland war unzureichend, Ende 2013 wird ein zweiter haircut notwendig. Der Euro wertet weiter ab, auf einen Wechselkurs von etwa 1/1 gegen den Dollar. Dies stärkt zwar die deutschen Exportbranchen, gleichzeitig werden aber die Importe, vor allem im Energiebereich, deutlich teurer. Die europäische Krise erfasst schließlich auch Deutschland, dessen Wirtschaft für die kommenden vier Jahre stagniert. Weitere globale Krisen, etwa der Einbruch der Binnennachfrage in China und mehrere Staatsstreiche im arabischen Raum, üben ebenfalls Druck auf das Wirtschaftswachstum auf. Der Zinsanstieg bleibt indes moderat, da Deutschland als sicherer Hafen für ausländische Anlagen gefragt bleibt. Deutschlands Bonität wird dennoch herabgestuft, da das Haushaltsdefizit auf rd. vier Prozent des BIP steigt. Die Bundestagswahl 2013 bringt im Ergebnis eine Große Koalition hervor, die sich über die notwendigen Schritte zur Haushaltskonsolidierung nicht einigen kann. Durch die Erfolge der „Alternative für Deutschland“ bei der Europa-Wahl 2014 und die anhaltende Euro-Krise verunsichert - die Target-Salden liegen inzwischen bei über einer Billion Euro - bereitet die Bundesregierung den Austritt aus der Gemeinschaftswährung vor. Großbritannien tritt 2017 nach einem Referendum ganz aus der EU aus. Institutionelle Änderungen am Haushaltsverfahren unterbleiben, der neue SPD-Finanzminister erhöht die Staatsausgaben und stellt die Haushaltskonsolidierung ein. ${ }^{38}$ Die Schuldenbremse wird mit Verweis die Regelung in Artikel 115 GG (,...im Falle von Naturkatastrophen oder außergewöhnlichen Notsituationen, die sich der Kontrolle des Staates entziehen...") vom Bundestag für die Jahre 2016 und 2017 ausgesetzt, die Schuldenquote steigt bis 2017 auf 90 Prozent des BIP.

Im Trendszenario $a$ bewahrheiten sich die Prognosen der Regierung und der Wirtschaftsforschungsinstitute mit gewissen Abstrichen, die den generell zu

38 Auf der Homepage der SPD gibt es keine Rubrik für „Öffentliche Finanzen“ mit den Themen Konsolidierung und Verschuldung (Stand: Juli 2013). Im SPD-Regierungsprogramm zur Bundestagswahl 2013 („Das Wir entscheidet“) findet sich nur eine Aussage zur Haushaltskonsolidierung, die wenig Engagement in diesem Themenfeld verspricht: „Die Konsolidierung der öffentlichen Haushalte rückt mit der Schuldenbremse stärker als bisher in den Mittelpunkt. Bund und Länder müssen ihren Beitrag dazu leisten.", ebd., 66. 
positiven Annahmen geschuldet sind. Jedoch zeigt die Regierung nur geringe Reformaktivität, Strukturveränderungen unterbleiben gänzlich. Die Wirtschaft wächst in den kommenden vier Jahren um durchschnittlich 1 Prozent, wobei eine zyklische Entwicklung zu beobachten ist. Die Arbeitslosigkeit bleibt im Wesentlichen konstant. Zwar entlastet die demographische Entwicklung den Arbeitsmarkt, doch sorgt das geringere Wirtschaftswachstum für nur wenige neue Arbeitsplätze. In Europa wird eine Politik des muddling through zur Norm. Nach dem Lissabon-Ansatz aus dem Jahr 2000 scheitert nun mit „Europa 2020“ auch die zweite Wachstumsstrategie der EU. Der Rückstand gegenüber den anderen globalen Wirtschaftsräumen tritt immer deutlicher zutage. ${ }^{39}$ Die Euro-Krise bleibt als Dauerthema erhalten, die Krisenländer bekommen ihr Verschuldungsproblem und ihre Strukturdefizit nicht in den Griff. Der Euro steht weiter unter einem Abwertungsdruck, was die Exporte beflügelt, und das Zinsniveau steigt nur geringfügig an, was die damit verbundene Entlastung des Bundeshaushalts verstetigt. Der Bundesregierung aus CDU/CSU und FDP gelingt es, den Haushalt zu stabilisieren, wobei die Annahmen des Finanzplans 2014 und 2015 dennoch überschritten werden. Das Defizitziel von 0,35 Prozent des BIP wird 2016 knapp erreicht. Die Gewerkschaften üben sich weiterhin in Lohnzurückhaltung, was die deutsche Wettbewerbsfähigkeit und damit den Export weiter stützt. Bei den endogenen politischen Faktoren sind hingegen keine Veränderungen oder Anstrengungen erkennbar, um den Verbindlichkeitsgrad der Haushaltsplanung oder die Stringenz des Budgetprozesses zu erhöhen. Der neue Finanzminister gibt sich mit der Erfüllung der grundgesetzlichen Vorgaben zufrieden, stärkere Konsolidierungsanstrengungen finden nicht statt. Die Schuldenquote liegt 2017 bei 73 Prozent des BIP.

Das Trendszenario $b$ schließlich unterstellt eine etwas positivere Entwicklung, wobei auch die Regierung etwas aktiver ans Werk geht als im Trendszenario $a$. Die Kreditaufnahme entwickelt sich etwa entlang der gegenwärtigen Prognose der Bundesregierung, das Wirtschaftswachstum liegt mit 1,2 Prozent im Jahresdurchschnitt etwas höher als im Trendszenario $a$. Allerdings fällt auch die Inflationsrate höher aus. Die Staatsschuldfinanzierung und lockere Geldpolitik der Notenbanken wirkt sich auf die Realmärkte aus, sodass die Teuerungsrate über dem Zielwert der EZB liegt. Die Zinsen bleiben wie in Sz. 1 auf einem niedrigen

39 Ein Vergleich der durchschnittlichen Wachstumsraten im OECD-Raum zeigt für die EU-15-Mitglieder gegenüber den Nicht-EU-Mitgliedern für den Zeitraum von 1995 bis 2010 einen durchschnittlichen jährlichen Rückstand von 0,7 Prozentpunkten. 
Niveau, trotz eines leichten Anstiegs. Der Euro ist ebenfalls weiter unter Abwertungsdruck. Die Bundesregierung versucht, die Probleme über eine intensivere internationale wie nationale Koordinierung in den Griff zu bekommen. Die auf europäischer Ebene 2011 implementierten Reformgesetze zur Verschärfung des europäischen Stabilitätspakts (six-pack) finden mehr Beachtung als die Vorgängerregelung. Insbesondere die Verschärfung des Verfahrens bei einem übermäBigen Defizit, der Aufbau eines Frühwarnsystems für Haushaltsnotlagen, eine bessere Haushaltsüberwachung und mehr Transparenz im Haushaltsverfahren zeigen erste Erfolge in den hoch verschuldeten Ländern. Die institutionellen Veränderungen bei der Einleitung eines Defizitverfahrens sowie die Schaffung einer makroökonomischen Koordinierung zum Abbau wirtschaftlicher Ungleichgewichte stärken die europäische Zusammenarbeit. Die EZB wird ebenfalls mehr in die wirtschaftspolitische Koordinierung eingebunden und stoppt ab dem Jahr 2015 den Ankauf von Staatsschuldtitel auf dem Sekundärmarkt. Auf nationaler Ebene wird die mittelfristige Finanzplanung als strategisches Konsolidierungsinstrument eingesetzt. Prognosen zur Wirtschafts- und Steuerentwicklung werden weitaus vorsichtiger als in der Vergangenheit getätigt, die unerwartet hohen Steuereinnahmen sowie geringere Zinszahlungen ausschließlich zur Tilgung verwendet. Der neue Finanzminister genießt die Rückendeckung der Bundeskanzlerin, die die Haushaltskonsolidierung in das Zentrum ihrer Regierungstätigkeit stellt. Im Ergebnis liegt die Schuldenquote im Jahr 2017 bei 69 Prozent des BIP.

\section{Zusammenfassung}

Ziel dieses Beitrages war die Abschätzung der zukünftigen Entwicklung der Verschuldung des Bundes und der Entwicklung des Haushaltes. Verschiedene Verfahren kamen zur Anwendung: die Abschätzung anhand von Prognosen des Domar-Modells, eine Überprüfung der mittelfristigen Finanzplanungen der Bundesregierung sowie Simulationen auf der Basis einer Regressionsanalyse. Schließlich wurden vier mögliche Ergebnisse auf der Grundlage der Szenariotechnik vorgestellt. Die Bandbreite der eingesetzten Szenarien zeigt, wie sehr die Ergebnisse/outcomes sowohl von endogenen Weichenstellungen als auch von exogenen Faktoren abhängig sind.

Das Domar-Modell lieferte die Prognose einer Schuldenquote von 70 bis 88 Prozent des BIP für das Jahr 2016. Die Finanzplanung geht - auf der Basis der vergangenen (sehr optimistischen) Schätzungen - von einer Nullverschuldung ab 2016 aus. Dies würde zu einer deutlichen Reduktion des Schuldenstandes führen. 
Ob diese Ziele tatsächlich erreicht werden, darf bezweifelt werden, zumal angesichts des bisher dokumentierten Zielerreichungsgrads solcher Planungen. Zusätzliche Probleme, wie etwa die implizite Staatsverschuldung, bleiben dabei ohnehin außen vor. Die Simulationen auf der Basis von Regressionsanalysen schließlich prognostizieren eher steigende Verschuldungsquoten. Bei diesen Modellen sind jedoch Sensitivitätsanalysen notwendig, auch hängt die Validität der zugrundeliegenden Modelle jeweils von der Zahl der einbezogenen Jahre und der Zahl der untersuchten Fälle ab.

Die untersuchten Szenarien wiesen dagegen in eine positivere Richtung; drei der vier diskutierten Entwicklungsmöglichkeiten würden zu einer Verbesserung gegenüber dem status quo führen. Allerdings liegt die Spannweite der Prognosen mit Blick auf die Schuldenquote für das Jahr 2017 bei etwa 30 Prozentpunkten des BIP, ein erkennbar hoher Wert. Die mittleren Trendszenarien, die deutlich realistischer erscheinen als die beiden Extremszenarien, ergeben in der Variante $a$ einen Wert von 73 Prozent und in der Variante $b$ einen Wert von 69 Prozent. Erst nach Einhaltung der Schuldenbremse ab 2016 wird die Schuldenstandsquote spürbar sinken. Die Diskussion der vier Szenarien zeigt aber auch, dass die beteiligten politischen Akteure mit Blick auf die endogenen Faktoren durchaus über Handlungsspielräume verfügen, mithin nicht nur auf Veränderungen der exogenen Faktoren reagieren sollten. 